\title{
Dynamic Programming, Optimal Control and Model Predictive Control
}

\author{
Lars Grüne
}

\begin{abstract}
In this chapter, we give a survey of recent results on approximate optimality and stability of closed loop trajectories generated by model predictive control (MPC). Both stabilizing and economic MPC are considered and both schemes with and without terminal conditions are analyzed. A particular focus of the chapter is to highlight the role dynamic programming plays in this analysis. As we will see, dynamic programming arguments are ubiquitous in the analysis of MPC schemes.
\end{abstract}

\section{Introduction}

Model Predictive Control (MPC), also known as Receding Horizon Control, is one of the most successful modern control techniques, both regarding its popularity in academics and its use in industrial applications [6, 10, 14, 28]. In MPC, the control input is synthesized via the repeated solution of finite horizon optimal control problems on overlapping horizons. Among the most fundamental properties to be investigated when analyzing MPC schemes are the stability and (approximate) optimality properties of the closed loop solutions generated by MPC. One interpretation of MPC is that an infinite horizon optimal control problem is split up into the repeated solution of auxiliary finite horizon problems [12].

Dynamic Programming (DP) is one of the fundamental mathematical techniques for dealing with optimal control problems [4, 5]. It provides a rule to split up a high (possibly infinite) dimensional optimization problem over a long (possibly infinite) time horizon into auxiliary optimization problems on shorter horizons, which are much easier to solve. While at a first glance this appears similar to the procedure just described for MPC, the approach is different, in the sense that in DP the exact information about the future of the optimal trajectories — by means of

Lars Grüne

Mathematical Institute, University of Bayreuth, 95440 Bayreuth, Germany, e-mail: lars.gruene@uni-bayreuth.de 
the corresponding optimal value function - is included in the auxiliary problem. Thus, it provides a characterization of the exact solution, at the expense that the auxiliary problems are typically difficult to formulate and the number of auxiliary problems becomes huge - the (in)famous "curse of dimensionality". In MPC, the future information is only approximated (for schemes with terminal conditions) or even completely disregarded (for schemes without terminal conditions). This makes the auxiliary problems easy to formulate and to solve and keeps the number of these problems low, but now at the expense that it does not yield an exact optimal solution of the original problem anymore.

However, it may still be possible that the solution trajectories generated by MPC are stable and approximately optimal, and the key for proving such statements is to make sure that the neglected future information only slightly affects the solution. The present chapter presents a survey of a selection of results in this direction and in particular shows that ideas from dynamic programming are essential for this purpose. As we will show, dynamic programming methods can be used for estimating near optimal performance under suitable conditions on the future information (Proposition 6 and Theorem 15 are examples for such statements) but also for ensuring that the future information satisfies these conditions (as, e.g., in Proposition 8 or Lemma 14(ii)). Moreover, dynamic programming naturally provides ways to derive stability or convergence from optimality via Lyapunov functions arguments, as in Proposition 3.

The chapter is organized as follows. In Section 2 we describe the setting and the MPC algorithm we consider in this chapter. Section 3 collects the results from dynamic programming we will need in the sequel. Section 4 then presents results for stabilizing MPC, in which the stage cost penalizes the distance to a desired equilibrium. Both schemes with and without terminal conditions are discussed. Section 5 extends this analysis to MPC schemes with more general stage costs, which is usually referred to as economic MPC. Section 6 concludes the chapter.

\section{Setting, definitions and notation}

In this chapter we consider discrete time optimal control problems of the form

Minimize $J_{N}\left(x_{0}, u\right)$ with respect to the control sequence $u$,

where $N \in \mathbb{N}_{\infty}:=\mathbb{N} \cup\{\infty\}$ and

$$
J_{N}\left(x_{0}, u\right)=\sum_{k=0}^{N-1} \ell(x(k), u(k)),
$$

subject to the dynamics and the initial condition

$$
x(k+1)=f(x(k), u(k)), \quad x(0)=x_{0}
$$


and the combined state and input constraints

$$
(x(k), u(k)) \in \mathbb{Y} \forall k=0, \ldots, N-1 \text { and } x(N) \in \mathbb{X}
$$

for all $k \in \mathbb{N}$ for which the respective values are defined. Here $\mathbb{Y} \subset X \times U$ is the constraint set, $X$ and $U$ are the the state and input value set, respectively, and $\mathbb{X}:=\{x \in X \mid \exists u \in U$ with $(x, u) \in \mathbb{Y}\}$ is the state constraint set. The sets $X$ and $U$ are metric spaces with metrics $d_{X}(\cdot, \cdot)$ and $d_{U}(\cdot, \cdot)$. Because there is no danger of confusion we usually omit the indices $X$ and $U$ in the metrics. We denote the solution of (2) by $x_{u}\left(k, x_{0}\right)$. Moreover, for the distance of a point $x \in X$ to another point $y \in X$ we use the short notation $|x|_{y}:=d(x, y)$.

For $x_{0} \in \mathbb{X}$ and $N \in \mathbb{N}$ we define the set of admissible control sequences as

$$
\mathbb{U}^{N}\left(x_{0}\right):=\left\{u \in U^{N} \mid\left(x_{u}\left(k, x_{0}\right), u(k)\right) \in \mathbb{Y} \forall k=0, \ldots, N-1 \text { and } x_{u}\left(N, x_{0}\right) \in \mathbb{X}\right\}
$$

and

$$
\mathbb{U}^{\infty}\left(x_{0}\right):=\left\{u \in U^{\infty} \mid\left(x_{u}\left(k, x_{0}\right), u(k)\right) \in \mathbb{Y} \forall k \in \mathbb{N}\right\}
$$

Since feasibility issues are not the topic of this chapter, we make the simplifying assumption that $\mathbb{U}^{N}\left(x_{0}\right) \neq \emptyset$ for all $x_{0} \in \mathbb{X}$ and all $N \in \mathbb{N}_{\infty}$. If desired, this assumption can be avoided using the techniques from, e.g., [9], [14, Chapter 7], [20, Chapter 5], or [27].

Corresponding to the optimal control problem (1) we define the optimal value function

$$
V_{N}\left(x_{0}\right):=\inf _{u \in \mathbb{U}^{N}\left(x_{0}\right)} J\left(x_{0}, u\right)
$$

and we say that a control sequence $u_{N}^{\star} \in \mathbb{U}^{N}\left(x_{0}\right)$ is optimal for initial value $x_{0} \in \mathbb{X}$ if $J\left(x_{0}, u_{N}^{\star}\right)=V_{N}\left(x_{0}\right)$ holds.

It is often desirable to solve optimal control problems with infinite horizon $N=\infty$, for instance because the control objective under consideration naturally leads to an infinite horizon problem (like stabilization or tracking problems) or because an optimal control is needed for an indefinite amount of time (as in many regulation problems). For such problems the optimal control is usually desired in feedback form, i.e., in the form $u_{N}^{\star}(k)=\mu(x(k))$ for a feedback map $\mu: \mathbb{X} \rightarrow \mathbb{U}$. Except for special cases like linear quadratic problems without constraints, computing infinite horizon optimal feedback laws is in general a very difficult task. On the other hand, very accurate approximations to optimal control sequences $u_{N}^{\star}$ for finite horizon problems, particularly with moderate $N$, can be computed easily and fast (sometimes within a few milliseconds), and often also reliably with state-of-theart numerical optimization routines, even for problems in which the dynamics (2) are governed by partial differential equations. The following Receding Horizon or Model Predictive Control algorithm (henceforth abbreviated by MPC) is therefore an attractive alternative to solving an infinite horizon optimal control problem. 


\section{Algorithm 1 (Basic Model Predictive Control Algorithm)}

(Step 0) Fix a (finite) optimization horizon $N \in \mathbb{N}$ and set $k:=0$; let an initial value $x_{M P C}(0)$ be given

(Step 1) Compute an optimal control sequence $u_{N}^{\star}$ of Problem (1) for $x_{0}=x_{M P C}(k)$

(Step 2) Define the MPC feedback law value $\mu_{N}\left(x_{M P C}(k)\right):=u_{N}^{\star}(0)$

(Step 3) Set $x_{M P C}(k+1):=f\left(x_{M P C}(k+1), \mu_{N}\left(x_{M P C}(k)\right)\right), k:=k+1$ and go to (Step 1)

We note that although derived from an open loop optimal control sequence $u_{N}^{\star}, \mu_{N}$ is indeed a map from $\mathbb{X}$ to $U$, however, it will in general not be given in the form of an explicit formula. Rather, given $x_{M P C}(k)$, the value $\mu_{N}\left(x_{M P C}(k)\right)$ is obtained by solving the optimal control problem in Step 1 of Algorithm 1, which is usually done numerically.

In MPC, one often introduces additional terminal conditions, consisting of a terminal constraint set $\mathbb{X}_{0} \subseteq \mathbb{X}$ and a terminal cost $F: \mathbb{X}_{0} \rightarrow \mathbb{R}$. To this end, the optimization objective $J_{N}$ is modified to

$$
J_{N}^{t c}(x, u)=\sum_{k=0}^{N-1} \ell(x(k), u(k))+F(x(N))
$$

and the last constraint in (3) is tightened to

$$
x(N) \in \mathbb{X}_{0}
$$

Moreover, we denote the corresponding space of admissible control sequences by

$$
\mathbb{U}_{0}^{N}\left(x_{0}\right):=\left\{u \in \mathbb{U}^{N}\left(x_{0}\right) \mid x_{u}\left(N, x_{0}\right) \in \mathbb{X}_{0}\right\}
$$

and the optimal value function by

$$
V_{N}^{t c}\left(x_{0}\right):=\inf _{u \in \mathbb{U}_{0}^{N}\left(x_{0}\right)} J\left(x_{0}, u\right)
$$

Observe that the problem without terminal conditions is obtained for $F \equiv 0$ and $\mathbb{X}_{0}=\mathbb{X}$.

Again, a control $u_{N}^{t c \star} \in \mathbb{U}_{0}^{N}\left(x_{0}\right)$ is called optimal if $V_{N}^{t c}\left(x_{0}\right)=J_{N}^{t c}\left(x_{0}, u_{N}^{t c \star}\right)$. Due to the terminal constraints it is in general not guaranteed that $\mathbb{U}_{0}^{N}\left(x_{0}\right) \neq \emptyset$ for all $x_{0} \in \mathbb{X}$. We therefore define $\mathbb{X}_{N}:=\left\{x_{0} \in \mathbb{X} \mid \mathbb{U}_{0}^{N}\left(x_{0}\right) \neq \emptyset\right\}$. For MPC in which $J_{N}^{t c}$ is minimized in Step 1 we denote the resulting feedback law by $\mu_{N}^{t c}$. Note that $\mu_{N}^{t c}$ is defined on $\mathbb{X}_{N}$.

A priori, it is not clear, at all, whether the trajectory $x_{M P C}$ generated by the MPC algorithm enjoys approximate optimality properties or qualitative properties like stability. In the remainder of this chapter, we will give conditions under which such properties can be guaranteed. In order to measure the optimality of the closed loop trajectory, we introduce its closed loop finite and infinite horizon values 


$$
J_{K}^{c l}\left(x, \mu_{N}\right):=\sum_{k=0}^{K-1} \ell\left(x_{M P C}(k), \mu_{N}\left(x_{M P C}(k)\right)\right)
$$

and

$$
J_{\infty}^{c l}\left(x, \mu_{N}\right):=\limsup _{K \rightarrow \infty} J_{K}^{c l}\left(x_{M P C}(0), \mu_{N}\right)
$$

where in both cases the initial value $x_{M P C}(0)=x$ is used.

\section{Dynamic programming}

Dynamic programming is a name for a set of relations between optimal value functions and optimal trajectories at different time instants. In what follows we state those relations which are important for the remainder of this chapter. For their proofs we refer to [14, Chapters 3 and 4].

For the finite horizon problem without terminal conditions the following equations and statements hold for all $N \in \mathbb{N}$ and all $K \in \mathbb{N}$ with $K \leq N$ (using $V_{0}(x) \equiv 0$ in case $K=N)$ :

$$
V_{N}(x)=\inf _{u \in \mathbb{U}^{K}(x)}\left\{J_{K}(x, u)+V_{N-K}\left(x_{u}(K, x)\right)\right\}
$$

If $u_{N}^{\star} \in \mathbb{U}^{N}(x)$ is an optimal control for initial value $x$ and horizon $N$, then

$$
V_{N}(x)=J_{K}\left(x, u_{N}^{\star}\right)+V_{N-K}\left(x_{u_{N}^{\star}}(K, x)\right)
$$

and

$$
\begin{aligned}
& \text { the sequence } u_{K}:=\left(u_{N}^{\star}(K), \ldots, u_{N}^{\star}(N-1)\right) \in \mathbb{U}^{N-K}\left(x_{u_{N}^{\star}}(K, x)\right) \\
& \text { is an optimal control for initial value } x_{u_{N}^{\star}}(K, x) \text { and horizon } N-K \text {. }
\end{aligned}
$$

Moreover, for all $x \in \mathbb{X}$ the MPC feedback law $\mu_{N}$ satisfies

$$
V_{N}(x)=\ell\left(x, \mu_{N}(x)\right)+V_{N-1}\left(f\left(x, \mu_{N}(x)\right)\right) .
$$

For the finite horizon problem with terminal conditions the following holds for all $N \in \mathbb{N}$ and all $K \in \mathbb{N}$ with $K \leq N$ (using $V_{0}^{t c}(x)=F(x)$ in case $K=N$ ):

$$
V_{N}^{t c}(x)=\inf _{u \in \mathbb{U}_{N-K}^{K}(x)}\left\{J_{K}(x, u)+V_{N-K}^{t c}\left(x_{u}(K, x)\right)\right\},
$$

where $\mathbb{U}_{N-K}^{K}\left(x_{0}\right):=\left\{u \in \mathbb{U}^{K}\left(x_{0}\right) \mid x_{u}\left(N, x_{0}\right) \in \mathbb{X}_{N-K}\right\}$. If $u_{N}^{t c \star} \in \mathbb{U}_{0}^{N}(x)$ is an optimal control for initial value $x$ and horizon $N$, then

$$
V_{N}^{t c}(x)=J_{K}\left(x, u_{N}^{t c \star}\right)+V_{N-K}^{t c}\left(x_{u_{N}^{t c \star}}(K, x)\right)
$$

and 
the sequence $u_{K}^{t c}:=\left(u_{N}^{t c \star}(K), \ldots, u_{N}^{t c \star}(N-1)\right) \in \mathbb{U}^{N-K}\left(x_{u_{N}^{t c \star}}(K, x)\right)$

is an optimal control for initial value $x_{u_{N}^{t c \star}}(K, x)$ and horizon $N-K$.

Moreover, for all $x \in \mathbb{X}$ the MPC feedback law $\mu_{N}^{t c}$ satisfies

$$
V_{N}^{t c}(x)=\ell\left(x, \mu_{N}^{t c}(x)\right)+V_{N-1}^{t c}\left(f\left(x, \mu_{N}^{t c}(x)\right)\right) .
$$

Finally, for the infinite horizon problem the following equations and statements hold for all $K \in \mathbb{N}$ :

$$
V_{\infty}(x)=\inf _{u \in \mathbb{U}^{K}(x)}\left\{J_{K}(x, u)+V_{\infty}\left(x_{u}(K, x)\right)\right\}
$$

If $u_{\infty}^{\star}$ is an optimal control for initial value $x$ and horizon $N$, then

$$
V_{\infty}(x)=J_{K}\left(x, u_{\infty}^{\star}\right)+V_{\infty}\left(x_{u_{\infty}^{\star}}(K, x)\right)
$$

and

$$
\begin{aligned}
& \text { the sequence } u_{K}:=\left(u_{\infty}^{\star}(K), u_{\infty}^{\star}(K+1), \ldots\right) \in \mathbb{U}^{\infty}\left(x_{u_{\infty}^{\star}}(K, x)\right) \\
& \text { is an optimal control for initial value } x_{u_{\infty}^{\star}}(K, x) .
\end{aligned}
$$

The equations just stated can be used as the basis of numerical algorithms, see, e.g., $[5,17]$ and the references therein. Here, however, we rather use them as tools for the analysis of the performance of the MPC algorithm. Besides the equalities, above, which refer to the optimal trajectories, we will also need corresponding inequalities. These will be used in order to estimate $J_{K}^{c l}$ and $J_{\infty}^{c l}$ as shown in the following proposition.

Proposition 2 Assume there is function $\varepsilon: \mathbb{X} \rightarrow \mathbb{R}$ such that the approximate dynamic programming inequality

$$
V_{N}(x)+\varepsilon(x) \geq \ell\left(x, \mu_{N}(x)\right)+V_{N}\left(f\left(x, \mu_{N}(x)\right)\right)
$$

holds for all $x \in \mathbb{X}$. Then for each MPC closed loop solution $x_{M P C}$ and all $K \in \mathbb{N}$ the inequality

$$
J_{K}^{c l}\left(x_{M P C}(0), \mu_{N}\right) \leq V_{N}\left(x_{M P C}(0)\right)-V_{N}\left(x_{M P C}(K)\right)+\sum_{k=0}^{K-1} \varepsilon_{k}
$$

holds for $\varepsilon_{k}=\varepsilon\left(x_{M P C}(k)\right)$. If, in addition, $\hat{\varepsilon}:=\limsup _{K \rightarrow \infty} \sum_{k=0}^{K-1} \varepsilon_{k}<\infty$ and $\liminf _{K \rightarrow \infty} V_{N}\left(x_{M P C}(K)\right) \geq 0$ hold, then also

$$
J_{\infty}^{c l}\left(x_{M P C}(0), \mu_{N}\right) \leq V_{N}\left(x_{M P C}(0)\right)+\hat{\varepsilon}
$$

holds. The same statements are true when $V_{N}$ and $\mu_{N}$ are replaced by their terminal conditioned counterparts $V_{N}^{t c}$ and $\mu_{N}^{t c}$, respectively.

Proof. Observing that $x_{M P C}(k+1)=f\left(x, \mu_{N}(x)\right)$ for $x=x_{M P C}(k)$ and using (15) with this $x$ we have 


$$
\begin{aligned}
J_{K}^{c l}\left(x_{M P C}(0), \mu_{N}\right) & =\sum_{k=0}^{K-1} \ell\left(x_{M P C}(k), \mu_{N}\left(x_{M P C}(k)\right)\right) \\
& \leq \sum_{k=0}^{K-1}\left[V_{N}\left(x_{M P C}(k)\right)-V_{N}\left(x_{M P C}(k+1)\right)+\varepsilon_{k}\right] \\
& =V_{N}\left(x_{M P C}(0)\right)-V_{N}\left(x_{M P C}(K)\right)+\sum_{k=0}^{K-1} \varepsilon_{k},
\end{aligned}
$$

which shows the first claim. The second claim follows from the first by taking the upper limit for $K \rightarrow \infty$. The proof for the terminal conditioned case is identical.

\section{Stabilizing MPC}

Using the dynamic programming results just stated, we will now derive estimates for $J_{\infty}^{c l}$ in the case of stabilizing MPC. Stabilizing MPC refers to the case in which the stage $\cos t \ell$ penalizes the distance to a desired equilibrium. More precisely, let $\left(x_{*}, u_{*}\right) \in \mathbb{Y}$ be an equilibrium, i.e., $f\left(x_{*}, u_{*}\right)=x_{*}$. Then throughout this section we assume that there is $\alpha_{1} \in \mathscr{K}_{\infty}$ such that ${ }^{1} \ell$ satisfies

$$
\ell\left(x_{*}, u_{*}\right)=0 \text { and } \ell(x, u) \geq \alpha_{1}\left(|x|_{x_{*}}\right)
$$

for all $x \in \mathbb{X}$. Moreover, for the terminal cost $F$ we assume

$$
F(x) \geq 0 \text { for all } x \in \mathbb{X}_{0} .
$$

We note that (18) trivially holds in case no terminal cost is used, i.e., if $F \equiv 0$.

The purpose of this choice of $\ell$ is to force the optimal trajectories - and thus hopefully also the MPC trajectories - to converge to $x_{*}$. The following proposition shows that this hope is justified under suitable conditions, where the approximate dynamic programming inequality (15) plays a pivotal role.

Proposition 3 Let the assumptions of Proposition 2, (17) and (18) (in case of terminal conditions) hold with $\varepsilon(x) \leq \eta \alpha_{1}\left(|x|_{x_{*}}\right)$ for all $x \in \mathbb{X}$ and some $\eta<1$. Then $x_{M P C}(k) \rightarrow x_{*}$ as $k \rightarrow \infty$.

Proof. We first observe that the assumptions imply $V_{N}(x) \geq 0$ or $V_{N}^{t c}(x) \geq 0$, respectively. We continue the proof for $V_{N}$, the proof for $V_{N}^{t c}$ is identical. Assume $x_{M P C}(k) \nrightarrow \rightarrow x_{*}$, i.e., there are $\delta>0$ and a sequence $k_{p} \rightarrow \infty$ with $\left|x_{M P C}\left(k_{p}\right)\right|_{x_{*}} \geq \delta$ for all $p \in \mathbb{N}$. Then by induction over (15) with $x=x_{M P C}(k)$ we get

${ }^{1}$ The space $\mathscr{K}_{\infty}$ consists of all functions $\alpha:[0, \infty) \rightarrow[0, \infty)$ with $\alpha(0)=0$ which are continuous, strictly increasing and unbounded. 


$$
\begin{aligned}
V_{N}\left(x_{M P C}(K)\right) & \leq V_{N}\left(x_{M P C}(0)\right)-\sum_{k=0}^{K-1}\left[\ell\left(x_{M P C}(k), \mu_{N}\left(x_{M P C}(k)\right)\right)-\varepsilon\left(x_{M P C}(k)\right)\right] \\
& \leq V_{N}\left(x_{M P C}(0)\right)-\sum_{k=0}^{K-1}(1-\eta) \alpha_{1}\left(\left|x_{M P C}(k)\right|_{x_{*}}\right) \\
& \leq V_{N}\left(x_{M P C}(0)\right)-\sum_{\substack{p \in \mathbb{N} \\
k p \leq K-1}}(1-\eta) \alpha_{1}\left(\left|x_{M P C}\left(k_{p}\right)\right|_{x_{*}}\right) \\
& \leq V_{N}\left(x_{M P C}(0)\right)-\#\left\{p \in \mathbb{N} \mid k_{p} \leq K\right\}(1-\eta) \alpha_{1}(\delta) .
\end{aligned}
$$

Now as $K \rightarrow \infty$ the number $\#\left\{p \in \mathbb{N} \mid k_{p} \leq K\right\}$ grows unboundedly, which implies that $V_{N}\left(x_{M P C}(K)\right)<0$ for sufficiently large $K$ which contradicts the non-negativity of $V_{N}$.

We remark that under additional conditions (essentially appropriate upper bounds on $V_{N}$ or $V_{N}^{t c}$, respectively), asymptotic stability of $x_{*}$ can also be established, see, e.g., [14, Theorem 4.11] or [28, Theorem 2.22].

\subsection{Terminal conditions}

In this section we use the terminal conditions in order to ensure that the approximate dynamic programming inequality (15) holds with $\varepsilon(x) \leq 0$ and $V_{N}^{t c}(x) \geq 0$. Then Proposition 2 applies and yields $J_{\infty}^{c l}\left(x_{M P C}(0), \mu_{N}^{t c}\right) \leq V_{N}^{t c}\left(x_{M P C}(0)\right)$ while Proposition 3 implies $x_{M P C}(k) \rightarrow x_{*}$. The key for making this approach work is the following assumption.

Assumption 4 For each $x \in \mathbb{X}$ there is $u_{x} \in U$ with $\left(x, u_{x}\right) \in \mathbb{Y}, f\left(x, u_{x}\right) \in \mathbb{X}$ and

$$
\ell\left(x, u_{x}\right)+F\left(f\left(x, u_{x}\right)\right) \leq F(x) .
$$

While conditions like Assumption 4 were already developed in the 1990s, e.g., in [7, 22, 25], it was the paper [23] published in 2000 which established this condition as the standard assumption for stabilizing MPC with terminal conditions. The particular case $\mathbb{X}=\left\{x_{*}\right\}$ was investigated in detail already in the 1980s in the seminal paper [19].

Theorem 5. Consider the MPC scheme with terminal conditions satisfying (17), (18) and Assumption 4. Then the inequality $J_{\infty}^{c l}\left(x, \mu_{N}^{t c}\right) \leq V_{N}^{t c}(x)$ and the convergence $x_{M P C}(k) \rightarrow x_{*}$ for $k \rightarrow \infty$ hold for all $x \in \mathbb{X}_{N}$ and the closed loop solution $x_{M P C}(k)$ with $x_{M P C}(0)=x$.

Proof. As explained before the theorem, it is sufficient to prove (15) with $\varepsilon(x) \leq 0$ and $V_{N}^{t c}(x) \geq 0$; then Propositions 2 and 3 yield the assertions. The inequality $V_{N}^{t c}(x) \geq$ 
0 is immediate from (17) and (18). For proving (15) with $\varepsilon(x) \leq 0$, using $u_{x}$ from Assumption 4 with $x=x_{u}\left(N-1, x_{0}\right)$ we get

$$
\begin{aligned}
V_{N-1}^{t c}\left(x_{0}\right) & =\inf _{u \in \mathbb{U}_{0}^{N-1}\left(x_{0}\right)} \sum_{k=0}^{N-2} \ell\left(x_{u}\left(k, x_{0}\right), u(k)\right)+F\left(x_{u}\left(N-1, x_{0}\right)\right) \\
& \geq \inf _{u \in \mathbb{U}_{0}^{N-1}\left(x_{0}\right)} \sum_{k=0}^{N-2} \ell\left(x_{u}\left(k, x_{0}\right), u(k)\right)+\ell\left(x, u_{x}\right)+F\left(f\left(x, u_{x}\right)\right) \\
& \geq \inf _{u \in \mathbb{U}_{0}^{N}\left(x_{0}\right)} \sum_{k=0}^{N-1} \ell\left(x_{u}\left(k, x_{0}\right), u(k)\right)+F\left(x_{u}\left(N, x_{0}\right)\right)=V_{N}^{t c}\left(x_{0}\right)
\end{aligned}
$$

Inserting this inequality for $x_{0}=f\left(x, \mu_{N}^{t c}(x)\right)$ into (11) we obtain

$$
V_{N}^{t c}(x)=\ell\left(x, \mu_{N}^{t c}(x)\right)+V_{N-1}^{t c}\left(f\left(x, \mu_{N}^{t c}(x)\right)\right) \geq \ell\left(x, \mu_{N}^{t c}(x)\right)+V_{N}^{t c}\left(f\left(x, \mu_{N}^{t c}(x)\right)\right)
$$

and thus (15) with $\varepsilon \equiv 0$.

A drawback of the inequality $J_{\infty}^{c l}\left(x, \mu_{N}^{t c}\right) \leq V_{N}^{t c}(x)$ is that it is in general quite difficult to give estimates for $V_{N}^{t c}(x)$. Under reasonable assumptions it can be shown that $V_{N}^{t c}(x) \rightarrow V_{\infty}(x)$ for $N \rightarrow \infty$ [14, Section 5.4]. This implies that the MPC solution is near optimal for the infinite horizon problem for $N$ sufficiently large. However, it is in general difficult to make statements about the speed of convergence of $V_{N}^{t c}(x) \rightarrow$ $V_{\infty}(x)$ as $N \rightarrow \infty$ and thus to estimate the length of the horizon $N$ which is needed for a desired degree of suboptimality.

\subsection{No terminal conditions}

The decisive property induced by Assumption 4 and exploited in the proof of Theorem 5 is the fact that $V_{N-1}^{t c}\left(x_{0}\right) \geq V_{N}^{t c}\left(x_{0}\right)$. Without this inequality, (11) implies that (15) with $\varepsilon \equiv 0$ cannot in general be satisfied. Without terminal conditions and under the condition (17) it is, however, straightforward to see that the opposite inequality $V_{N-1}^{t c}\left(x_{0}\right) \leq V_{N}^{t c}\left(x_{0}\right)$ holds, where in most cases this inequality is strict. This means that without terminal conditions we need to work with positive $\varepsilon$. The following proposition, which was motivated by a similar "relaxed dynamic programming" inequality used in [21], introduces a variant of Proposition 2 which we will use for this purpose.

Proposition 6 Assume there is a constant $\alpha \in(0,1]$ such that the relaxed dynamic programming inequality

$$
V_{N}(x) \geq \alpha \ell\left(x, \mu_{N}(x)\right)+V_{N}\left(f\left(x, \mu_{N}(x)\right)\right)
$$

holds for all $x \in \mathbb{X}$. Then for each MPC closed loop solution $x_{M P C}$ and all $K \in \mathbb{N}$ the inequality 


$$
J_{\infty}^{c l}\left(x_{M P C}(0), \mu_{N}\right) \leq V_{\infty}\left(x_{M P C}(0)\right) / \alpha
$$

and, if additionally (17) holds, the convergence $x_{M P C}(k) \rightarrow x_{*}$ for $k \rightarrow \infty$ hold.

Proof. Applying Proposition 2 with $\varepsilon(x)=(1-\alpha) \ell\left(x, \mu_{N}(x)\right)$ yields

$$
\begin{aligned}
J_{K}^{c l}\left(x_{M P C}(0), \mu_{N}\right) \leq & V_{N}\left(x_{M P C}(0)\right)-V_{N}\left(x_{M P C}(K)\right) \\
& +(1-\alpha) \underbrace{\sum_{k=0}^{K-1} \ell\left(x_{M P C}(k), \mu_{N}\left(x_{M P C}(k)\right)\right)}_{=J_{K}^{c l}\left(x_{M P C}(0), \mu_{N}\right)} .
\end{aligned}
$$

Using $V_{N} \geq 0$ this implies $\alpha J_{K}^{c l}\left(x_{M P C}(0), \mu_{N}\right) \leq V_{N}\left(x_{M P C}(0)\right)$ which implies the first assertion by letting $K \rightarrow \infty$ and dividing by $\alpha$. The convergence $x_{M P C}(k) \rightarrow x_{*}$ follows from Proposition 3.

A simple condition under which we can guarantee that (19) holds is given in the following assumption.

Assumption 7 There are constants $\gamma_{k}>0, k \in \mathbb{N}$ with $\sup _{k \in \mathbb{N}} \gamma_{k}<\infty$ and

$$
V_{k}(x) \leq \gamma_{k} \inf _{u \in U,(x, u) \in \mathbb{Y}} \ell(x, u) .
$$

A sufficient condition for Assumption 7 to hold is that $\ell$ is a polynomial satisfying (17) and the system can be controlled to $x_{*}$ exponentially fast. However, via an appropriate choice of $\ell$ Assumption 7 can also be satisfied if the system is not exponentially controllable, see, e.g., [14, Example 6.7].

The following theorem, taken with modifications from [29], shows that Assumption 7 implies (19).

Proposition 8 Consider the MPC scheme without terminal conditions satisfying Assumption 7. Then (19) holds with $\alpha=1-\left(\gamma_{2}-1\right)\left(\gamma_{N}-1\right) \prod_{k=0}^{N-1}\left(\frac{\gamma_{k}-1}{\gamma_{k}}\right)$.

Proof. First note that for $x=x_{*}$ (19) always holds because all expressions vanish. For $x \neq x_{*}$, we consider the MPC solution $x_{M P C}(\cdot)$ with $x_{M P C}(0)=x$, abbreviate $\lambda_{k}=\ell\left(x_{u_{N}^{\star}}(k, x), u_{N}^{\star}(k)\right)$ with $u_{N}^{\star}$ denoting the optimal control for initial value $x_{0}=x$, and $v=V_{N}\left(f\left(x, \mu_{N}(x)\right)\right)=V_{N}\left(x_{M P C}(1)\right)$. Then (19) becomes

$$
\sum_{k=0}^{N-1} \lambda_{k}-v \geq \alpha \lambda_{0}
$$

We prove the theorem by showing the inequality

$$
\lambda_{N-1} \leq\left(\gamma_{N}-1\right) \prod_{k=2}^{N-1}\left(\frac{\gamma_{k}-1}{\gamma_{k}}\right) \lambda_{0}
$$


for all feasible $\lambda_{0}, \ldots, \lambda_{N-1}$. From this (20) follows since the dynamic programming equation (4) with $x=x_{M P C}(1)$ and $K=N-2$ implies

$$
v \leq \sum_{n=1}^{N-2} \ell\left(x_{u_{N}^{\star}}(n, x), u_{N}^{\star}(n)\right)+V_{2}\left(x_{u_{N}^{\star}}(N-1, x)\right) \leq \sum_{n=1}^{N-2} \lambda_{n}+\gamma_{2} \lambda_{N-1}
$$

and thus (21), $\gamma_{2} \geq 1$ and $\lambda_{0}=1$ yield

$$
\sum_{n=0}^{N-1} \lambda_{n}-v \geq \lambda_{0}+\left(1-\gamma_{2}\right) \lambda_{N-1} \geq \lambda_{0}-\left(\gamma_{2}-1\right)\left(\gamma_{N}-1\right) \prod_{k=2}^{N-1}\left(\frac{\gamma_{k}-1}{\gamma_{k}}\right) \lambda_{0}=\alpha \lambda_{0} .
$$

i.e., (20). In order to prove (21), we start by observing that since $u_{K}:=\left(u_{N}^{\star}(K), \ldots\right.$, $\left.u_{N}^{\star}(N-1)\right)$ is an optimal control for initial value $x_{u_{N}^{\star}}(K, x)$ and horizon $N-K$, we obtain $\sum_{k=p}^{N-1} \lambda_{k}=V_{N-p}\left(x_{u_{N}^{\star}}(p+1)\right) \leq \gamma_{N-p} \lambda_{p}$, which implies

$$
\sum_{k=p+1}^{N-1} \lambda_{k} \leq\left(\gamma_{N-p}-1\right) \lambda_{p}
$$

for $p=0, \ldots, N-2$. From this we can conclude

$$
\lambda_{p}+\sum_{k=p+1}^{N-1} \lambda_{k} \geq \frac{\sum_{k=p+1}^{N-1} \lambda_{k}}{\gamma_{N-p}-1}+\sum_{k=p+1}^{N-1} \lambda_{k}=\frac{\gamma_{N-p}}{\gamma_{N-p}-1} \sum_{k=p+1}^{N-1} \lambda_{k} .
$$

Using this inequality inductively for $p=1, \ldots, N-2$ yields

$$
\sum_{k=1}^{N-1} \lambda_{k} \geq \prod_{k=1}^{N-2}\left(\frac{\gamma_{N-k}}{\gamma_{N-k}-1}\right) \lambda_{N-1}=\prod_{k=2}^{N-1}\left(\frac{\gamma_{k}}{\gamma_{k}-1}\right) \lambda_{N-1}
$$

Using (22) for $p=0$ we then obtain

$$
\left(\gamma_{N}-1\right) \lambda_{0} \geq \sum_{k=1}^{N-1} \lambda_{k} \geq \prod_{k=2}^{N-1}\left(\frac{\gamma_{k}}{\gamma_{k}-1}\right) \lambda_{N-1}
$$

which implies (21).

This proposition immediately leads to the following theorem.

Theorem 9. Consider the MPC scheme without terminal conditions satisfying Assumption 7. Then for all sufficiently large $N \in \mathbb{N}$ the inequality $J_{\infty}^{c l}\left(x, \mu_{N}\right) \leq V_{\infty}(x) / \alpha$ and the convergence $x_{M P C}(k) \rightarrow x_{*}$ for $k \rightarrow \infty$ hold for all $x \in \mathbb{X}$ and the closed loop solution $x_{M P C}(k)$ with $x_{M P C}(0)=x$, with $\alpha$ from Proposition 8 .

Proof. Since $\gamma_{\infty}:=\sup _{k \in \mathbb{N}} \gamma_{k}<\infty$ it follows that $\left(\gamma_{k}-1\right) / \gamma_{k} \leq\left(\gamma_{\infty}-1\right) / \gamma_{\infty}<1$ for all $k \in \mathbb{N}$, implying that $\alpha$ from Proposition 8 satisfies $\alpha \in(0,1]$ for sufficiently large $N$. For these $N$ the assertion follows from Proposition 6. 
We note that $\alpha$ from Proposition 8 is not optimal. In [15] (see also [30] and [14, Chapter 6]) the optimal bound

$$
\alpha=1-\frac{\left(\gamma_{N}-1\right) \prod_{k=2}^{N}\left(\gamma_{k}-1\right)}{\prod_{k=2}^{N} \gamma_{k}-\prod_{k=2}^{N}\left(\gamma_{k}-1\right)}
$$

is derived, however, at the expense of a much more involved proof than that of Proposition 8. The difference between the two bounds can be illustrated if we assume $\gamma_{k}=\gamma$ for all $k \in \mathbb{N}$ and compute the minimal $N \in \mathbb{N}$ such that $\alpha>0$ holds, i.e., the minimal $N$ for which Theorem 9 ensures the convergence $x_{M P C}(k) \rightarrow x_{*}$. For $\alpha$ from Proposition 8 we obtain the condition $N>2+2 \ln (\gamma-1) /(\ln \gamma-\ln (\gamma-1))$ while for $\alpha$ from (23) we obtain $N>2+\ln (\gamma-1) /(\ln \gamma-\ln (\gamma-1))$. The optimal $\alpha$ hence reduces the estimate for $N$ roughly by a factor of 2 .

The analysis can be extended to the situation in which $\alpha$ in (19) cannot be found for all $x \in \mathbb{X}$. In this case, one can proceed similarly as in the discussion after Theorem 15 , below, in order to obtain practical asymptotic stability, i.e., inequality (34), on bounded subsets of $\mathbb{X}$.

\section{Economic MPC}

Economic MPC has become the common name for MPC schemes in which the stage cost $\ell$ does not penalize the distance to an equilibrium $x_{*}$ which was determined a priori. Rather, $\ell$ models economic objectives, like high output, low energy consumption etc. or a combination thereof.

For such general $\ell$ many of the arguments from the previous section do not work for several reasons. First, the cost $J_{N}$ and thus the optimal value function $V_{N}$ is not necessarily nonnegative, a fact which was exploited in several places in the proofs in the last section. Second, the infinite sum in the infinite horizon objective need not converge and thus it may not make sense to talk about infinite horizon performance. Finally, optimal trajectories need not stay close or converge to an equilibrium, again a fact that was used in various places in the last section.

A systems theoretic property which effectively serves as a remedy for all these difficulties is contained in the following definition.

Definition 10 (Strict Dissipativity and Dissipativity) We say that an optimal control problem with stage cost $\ell$ is strictly dissipative at an equilibrium $\left(x^{e}, u^{e}\right) \in \mathbb{Y}$ if there exists a storage function $\lambda: \mathbb{X} \rightarrow \mathbb{R}$ bounded from below and satisfying $\lambda\left(x^{e}\right)=0$, and a function $\rho \in \mathscr{K}_{\infty}$ such that for all $(x, u) \in \mathbb{Y}$ the inequality

$$
\ell(x, u)-\ell\left(x^{e}, u^{e}\right)+\lambda(x)-\lambda(f(x, u)) \geq \rho\left(|x|_{x^{e}}\right)
$$

holds. We say that an optimal control problem with stage cost $\ell$ is dissipative at $\left(x^{e}, u^{e}\right)$ if the same conditions hold with $\rho \equiv 0$. 
We note that the assumption $\lambda\left(x^{e}\right)=0$ can be made without loss of generality because adding a constant to $\lambda$ does not invalidate (24).

The observation that strict dissipativity is the "right" property in order to analyze economic MPC schemes was first made by Diehl, Amrit and Rawlings in [8], where strict duality, i.e., strict dissipativity with a linear storage function, was used. The extension to the nonlinear notion of strict dissipativity was then made by Angeli and Rawlings in [3]. Although recent studies show that for certain classes of systems this property can be further (slightly) relaxed (see [26]), here we work with this condition because it provides a mathematically elegant way for dealing with economic MPC.

Remark 11 Strict dissipativity implies several important properties:

(i) The equilibrium $\left(x^{e}, u^{e}\right) \in \mathbb{Y}$ from Definition 10 is a strict optimal equilibrium in the sense that $\ell\left(x^{e}, u^{e}\right)<\ell(x, u)$ for all other admissible equilibria of $f$, i.e., all other $(x, u) \in \mathbb{Y}$ with $f(x, u)=x$. This follows immediately from (24).

(ii) The optimal equilibrium $x^{e}$ has the turnpike property, i.e., the following holds: For each $\delta>0$ there exists $\sigma_{\delta} \in \mathscr{L}$ such that ${ }^{2}$ for all $N, P \in \mathbb{N}, x \in \mathbb{X}$ and $u \in$ $\mathbb{U}^{N}(x)$ with $J_{N}^{u c}(x, u) \leq N \ell\left(x^{e}, u^{e}\right)+\delta$, the set $\mathscr{Q}(x, u, P, N):=\{k \in\{0, \ldots, N-$ $\left.1\}\left.|| x_{u}(k, x)\right|_{x^{e}} \geq \sigma_{\delta}(P)\right\}$ has at most $P$ elements. A proof of this fact can be found, e.g., in [14, Proposition 8.15]. The same property holds for all near optimal trajectories of the infinite horizon problem, provided it is well defined, cf. [14, Proposition 8.18].

(iii) If we define the modified or rotated cost $\tilde{\ell}(x, u):=\ell(x, u)-\ell\left(x^{e}, u^{e}\right)+\lambda(x)-$ $\lambda(f(x, u))$, then this modified cost satisfies (17), i.e., the basic property we exploited in the previous section.

The third property enables us to use the optimal control problem with modified $\operatorname{cost} \tilde{\ell}$ as an auxiliary problem in our analysis. The way this auxiliary problem is used crucially depends on whether we use terminal conditions or not. We start with the case with terminal conditions. Throughout this section, we assume that all functions under consideration are continuous in $x^{e}$.

\subsection{Terminal conditions}

For the economic MPC problem with terminal conditions we make exactly the same assumption on the terminal constraint set $\mathbb{X}_{0}$ and the terminal cost $F$ as in the stabilizing case, i.e., we again use Assumption 4 . We assume without loss of generality that $F\left(x^{e}\right)=0$, which implies that $F$ may attain negative values, because $\ell$ may be negative, too.

${ }^{2}$ The space $\mathscr{L}$ contains all functions $\sigma:[0, \infty) \rightarrow[0, \infty)$ which are continuous and strictly decreasing with $\lim _{t \rightarrow \infty} \sigma(t)=0$. 
Now the main trick - taken from [1] - lies in the fact that we introduce an adapted terminal cost for the problem with the modified cost $\tilde{\ell}$. To this end, we define the terminal cost $\widetilde{F}(x):=F(x)+\lambda(x)$. We denote the cost functional for the modified problems without and with terminal conditions by $\widetilde{J}_{N}$ and $\widetilde{J}_{N}^{t c}$, respectively, and the corresponding optimal value functions by $\widetilde{V}_{N}$ and $\widetilde{V}_{N}^{t c}$. Then a straightforward computation reveals that

$$
\widetilde{J}_{N}^{t c}(x, u)=J_{N}^{t c}(x, u)+\lambda(x)-N \ell\left(x^{e}, u^{e}\right),
$$

which means that the original and the modified optimization objective only differ in terms which do not depend on $u$. Hence, the optimal trajectories corresponding to $\widetilde{V}_{N}^{t c}$ and $V_{N}^{t c}$ coincide and the MPC scheme using the modified costs $\tilde{\ell}$ and $\widetilde{F}$ yields exactly the same closed loop trajectories as the scheme using $\ell$ and $F$.

One easily sees that $\widetilde{F}$ and $\tilde{\ell}$ also satisfy Assumption 4, i.e., that for each $x \in \mathbb{X}_{0}$ there is $u_{x} \in U$ with $\left(x, u_{x}\right) \in \mathbb{Y}, f\left(x, u_{x}\right) \in \mathbb{X}_{0}$ and

$$
\tilde{\ell}\left(x, u_{x}\right)+\widetilde{F}\left(f\left(x, u_{x}\right)\right) \leq \widetilde{F}(x)
$$

if $\ell$ and $F$ satisfy this property.

Moreover, if $\widetilde{F}$ is bounded on $\mathbb{X}_{0}$, then (26) implies $\widetilde{F}(x) \geq 0$ for all $x \in \mathbb{X}_{0}$. In order to see this, assume $F\left(x_{0}\right)<0$ for some $x_{0} \in \mathbb{X}_{0}$ and consider the control sequence defined by $u(k)=u_{x}$ with $u_{x}$ from (26) for $x=x_{u}\left(k, x_{0}\right)$. Then, $\tilde{\ell} \geq 0$ implies $F\left(x_{u}(k, x)\right) \leq F\left(x_{0}\right)<0$ for all $k \in \mathbb{N}$. Moreover, similar as in the proof of Theorem (3), the fact that $\tilde{\ell}$ satisfies (17) implies that $x_{u}\left(k, x_{0}\right) \rightarrow x^{e}$, because otherwise $F\left(x_{u}\left(k, x_{0}\right)\right) \rightarrow-\infty$ which contradicts the boundedness of $F$. But then continuity of $F$ in $x^{e}$ implies

$$
F\left(x^{e}\right)=\lim _{k \rightarrow \infty} F\left(x_{u}\left(k, x_{0}\right)\right) \leq F\left(x_{0}\right)<0
$$

which contradicts $F\left(x^{e}\right)=0$. Hence $\widetilde{F}(x) \geq 0$ follows for all $x \in \mathbb{X}_{0}$ (for a more detailed proof see [14, Proof of Theorem 8.13]).

As a consequence, the problem with the modified costs $\tilde{\ell}$ and $\widetilde{F}$ satisfies all the properties we assumed for the results in Section 4.1. Hence, Theorem 5 applies and yields the convergence $x_{M P C}(k) \rightarrow x^{e}$ and the performance estimate

$$
\widetilde{J}_{\infty}^{c l}\left(x, \mu_{N}^{t c}\right) \leq \widetilde{V}_{N}^{t c}(x) .
$$

As in the stabilizing case, under suitable conditions we obtain $\widetilde{V}_{N}^{t c}(x) \rightarrow \widetilde{V}_{\infty}(x)$ as $N \rightarrow \infty$. However, this only gives an estimate for the modified objective $\widetilde{J}_{\infty}^{c l}$ with stage cost $\tilde{\ell}$ but not for the original objective $J_{\infty}^{c l}$ with stage cost $\ell$.

In order to obtain an estimate for $J_{\infty}^{c l}$, one can proceed in two different ways: either one assumes $\ell\left(x^{e}, u^{e}\right)=0$ (which can always be achieved by adding $\ell\left(x^{e}, u^{e}\right)$ to $\ell$ ) and that the infinite horizon problem is well defined, which in particular means that $\left|V_{\infty}(x)\right|$ is finite. Then, from the definition of the problems, one sees that the relations 


$$
\widetilde{J}_{\infty}^{l}\left(x, \mu_{N}^{t c}\right)=J_{\infty}^{c l}\left(x, \mu_{N}^{t c}\right)-\lim _{k \rightarrow \infty} \lambda\left(x_{M P C}(k)\right)
$$

and

$$
\widetilde{V}_{\infty}(x) \leq V_{\infty}^{c l}(x)-\lim _{k \rightarrow \infty} \lambda\left(x_{u_{\infty}^{\star}}(k, x)\right) \text { and } V_{\infty}(x) \leq \widetilde{V}_{\infty}^{c l}(x)+\lim _{k \rightarrow \infty} \lambda\left(x_{\tilde{u}_{\infty}^{\star}}(k, x)\right)
$$

hold for $x_{M P C}(0)=x$ and $\tilde{u}_{\infty}^{\star}$ and $u_{\infty}^{\star}$ denoting the optimal controls corresponding to $\widetilde{V}_{\infty}(x)$ and $V_{\infty}(x)$, respectively.

Now strict dissipativity implies $x_{\tilde{u}_{\infty}^{\star}}(k, x) \rightarrow x^{e}$ and $x_{u_{\infty}^{\star}}(k, x) \rightarrow x^{e}$ as $k \rightarrow \infty$ (for details see [14, Proposition 8.18]), moreover, we already know that $x_{M P C}(k) \rightarrow x^{e}$ as $k \rightarrow \infty$. Since $\lambda\left(x^{e}\right)=0$ and $\lambda$ is continuous in $x^{e}$ this implies

$$
J_{\infty}^{c l}\left(x, \mu_{N}^{t c}\right) \rightarrow V_{\infty}(x)
$$

as $N \rightarrow \infty$, i.e., near optimal infinite horizon performance of the MPC closed loop for sufficiently large $N$.

The second way to obtain an estimate is to look at $J_{K}^{c l}\left(x, \mu_{N}^{t c}\right)$, which avoids setting $\ell\left(x^{e}, u^{e}\right)=0$ and making assumptions on $\left|V_{\infty}\right|$. However, while $x_{M P C}(k) \rightarrow x^{e}$, in the economic MPC context - even in the presence of strict dissipativity - the optimal trajectory $x_{u_{N}^{\star}}(k, x)$ will in general not end near $x^{e}$, see, e.g., the examples in $[11,12]$ or $\left[14\right.$, Chapter 8]. Hence, comparing $J_{K}^{c l}\left(x, \mu_{N}^{t c}\right)$ and $V_{K}(x)$ will in general not be meaningful. However, if for $x=x_{M P C}(0)$ we set $\delta(k):=\left|x_{M P C}(k)\right|_{x^{e}}$ and define the class of controls

$$
\mathbb{U}_{\delta(K)}^{K}(x):=\left\{\left.u \in \mathbb{U}^{K}(x)|| x_{u}(K, x)\right|_{x^{e}} \leq \delta(K)\right\}
$$

then it makes sense to compare $J_{K}^{c l}\left(x, \mu_{N}^{t c}\right)$ and $\inf _{u \in \mathbb{U}_{\delta(K)}^{K}(x)} J_{K}(x, u)$. More precisely, in [13] (see also [14, Section 8.4]) it was shown that there are error terms $\delta_{1}(N)$ and $\delta_{2}(K)$, converging to 0 as $N \rightarrow \infty$ or $K \rightarrow \infty$, respectively, such that the estimate

$$
J_{K}^{c l}\left(x, \mu_{N}^{t c}\right) \leq \inf _{u \in \mathbb{U}_{\delta(K)}^{K}(x)} J_{K}(x, u)+\delta_{1}(N)+\delta_{2}(K)
$$

holds. In other words, among all solutions steering $x$ into the $\delta(K)$-neighborhood of the optimal equilibrium $x^{e}$, MPC yields the cheapest one up to error terms vanishing as $K$ and $N$ become large.

In summary, except for inequality (28) which requires additional arguments, by using terminal conditions the analysis of economic MPC schemes is not much more difficult than the analysis of stabilizing MPC schemes. However, in contrast to the stabilizing case, so far no systematic procedure for the construction of terminal costs and constraint sets satisfying (26) is known. Hence, it appears attractive to avoid the use of terminal conditions. 


\subsection{No terminal conditions}

If we want to avoid the use of terminal conditions, the analysis becomes considerably more involved. The reason is that without terminal conditions the relation (25) changes to

$$
\widetilde{J}_{N}(x, u)=J_{N}(x, u)+\lambda(x)-\lambda\left(x_{u}(N, x)\right)-N \ell\left(x^{e}, u^{e}\right) .
$$

This means that the difference between $J_{N}$ and $\widetilde{J}_{N}$ now depends on $u$ and consequently the optimal trajectories do no longer coincide. Moreover, the central property exploited in the proof of Proposition 8, whose counterpart in the setting of this section would be that $\lambda_{N-1}=\ell\left(x_{u_{N}^{\star}}(N-1, x), u_{N}^{\star}(N-1)\right)$ is close to $\ell\left(x^{e}, u^{e}\right)$, is in general not true for economic MPC, not even for simple examples, see $[11,12]$ or [14, Chapter 8]. Hence, we cannot expect the arguments from the stabilizing case to work.

For these reasons, we have to use different arguments, which are combinations of arguments found in $[11,12,18]$. To this end we make the following assumptions.

Assumption 12 (i) The optimal control problem is strictly dissipative in the sense of Definition 10.

(ii) There exist functions $\gamma_{V}, \gamma_{\tilde{V}}$ and $\gamma_{\lambda} \in \mathscr{K}_{\infty}$ as well as $\omega, \tilde{\omega} \in \mathscr{L}$ such that the following inequalities hold for all $x \in \mathbb{X}$ and all $N \in \mathbb{N}_{\infty}$ :

$$
\begin{aligned}
\text { (a) } & \left|V_{N}(x)-V_{N}\left(x^{e}\right)\right| & \leq \gamma_{V}\left(|x|_{x^{e}}\right)+\omega(N) \\
\text { (b) } & \left|\widetilde{V}_{N}(x)-\widetilde{V}_{N}\left(x^{e}\right)\right| & \leq \gamma_{\widetilde{V}}\left(|x|_{x^{e}}\right)+\widetilde{\omega}(N) \\
\text { (c) } & \left|\lambda(x)-\lambda\left(x^{e}\right)\right| & \leq \gamma_{\lambda}\left(|x|_{x^{e}}\right)
\end{aligned}
$$

Part (ii) of this assumption is a uniform continuity assumption in $x^{e}$. For the optimal value functions $V_{N}$ and $\widetilde{V}_{N}$ it can, e.g., be guaranteed by local controllability around $x^{e}$, see [11, Theorem 6.4]. We note that this assumption together with the obvious inequality $V_{N}\left(x^{e}\right) \leq N \ell\left(x^{e}, u^{e}\right)$ and boundedness of $\mathbb{X}$ implies $V_{N}(x) \leq N \ell\left(x^{e}, u^{e}\right)+\delta$ with $\delta=\sup _{x \in \mathbb{X}} \gamma_{V}\left(|x|_{x^{e}}\right)+\omega(0)$. Hence, the optimal trajectories have the turnpike property according to Remark 11(ii).

For writing (in)equalities that hold up to an error term, we use the following convenient notation: for a sequence of functions $a_{J}: \mathbb{X} \rightarrow \mathbb{R}, J \in \mathbb{N}$, and another function $b: \mathbb{X} \rightarrow \mathbb{R}$ we write $a_{J}(x) \approx_{J} b(x)$ if $\lim _{J \rightarrow \infty} \sup _{x \in \mathbb{X}} a_{J}(x)-b(x)=0$ and we write $a_{J}(x) \lesssim_{J} b(x)$ if $\limsup _{J \rightarrow \infty} \sup _{x \in \mathbb{X}} a_{J}(x)-b(x) \leq 0$. In words, $\approx_{J}$ means "= up to terms which are independent of $x$ and vanish as $J \rightarrow \infty "$, and $\lesssim_{J}$ means the same for $\leq$.

With these assumptions and notation we can now prove the following relations. For simplicity of exposition in what follows we limit ourselves to a bounded state space $\mathbb{X}$. If this is not satisfied, the following considerations can be made for bounded subsets of $\mathbb{X}$. As we will see, dynamic programming arguments are ubiquitous in the following considerations. 
Lemma 13 Let $\mathbb{X}$ be bounded. Then under Assumptions 12 the following approximate equalities hold.

$$
\begin{array}{rlll}
V_{N}(x) & \approx_{S} & J_{M}\left(x, u_{N}^{\star}\right)+V_{N-M}\left(x^{e}\right) & \text { for all } M \notin \mathscr{Q}\left(x, u_{N}^{\star}, P, N\right) \\
V_{N}\left(x^{e}\right) & \approx_{S} & M \ell\left(x^{e}, u^{e}\right)+V_{N-M}\left(x^{e}\right) & \text { for all } M \notin \mathscr{Q}\left(x^{e}, u_{N}^{\star e}, P, N\right) \\
\widetilde{V}_{N}(x) & \approx_{N} & V_{N}(x)+\lambda(x)-V_{N}\left(x^{e}\right) &
\end{array}
$$

Here $P \in \mathbb{N}$ is an arbitrary number, $S:=\min \{P, N-M\}$, $u_{N}^{\star}$ is the control minimizing $J_{N}(x, u), u_{N}^{\star e}$ is the control minimizing $J_{N}\left(x^{e}, u\right)$, and $\mathscr{Q}$ is the set from Remark 11(ii). Moreover, $(i)$ and (ii) also apply to the optimal control problem with stage cost $\tilde{\ell}$.

Proof. (i) Observe that using the constant control $u \equiv u^{e}$ we can estimate $V_{N}\left(x^{e}\right) \leq$ $J_{N}\left(x^{e}, u\right)=N \ell\left(x^{e}, u^{e}\right)$. Thus, using Assumption 12 we get $J_{N}\left(x, u_{N}^{\star}\right) \leq N \ell\left(x^{e}, u^{e}\right)+$ $\gamma_{V}\left(|x|_{x^{e}}\right)+\omega(N)$, hence the turnpike property from Remark 11(ii) applies to the optimal trajectory with $\delta=\gamma_{V}\left(|x|_{x^{e}}\right)+\omega(N)$. This in particular ensures $\left|x_{u_{N}^{\star}}(M, x)\right|_{x^{e}} \leq$ $\sigma_{\delta}(P)$ for all $M \notin \mathscr{Q}\left(x, u_{N}^{\star}, P, N\right)$.

Now the dynamic programming equation (5) yields

$$
V_{N}(x)=J_{M}\left(x, u_{N}^{\star}\right)+V_{N-M}\left(x_{u_{N}^{\star}}(M, x)\right) .
$$

Hence, (i) holds with remainder terms $R_{1}(x, M, N)=V_{N-M}\left(x_{u_{N}^{\star}}(M, x)\right)-V_{N-M}\left(x^{e}\right)$. For any $P \in \mathbb{N}$ and any $M \notin \mathscr{Q}\left(x, u_{N}^{\star}, P, N\right)$ we have $\left|R_{1}(x, M, N)\right| \leq \gamma_{V}\left(\left|x_{u_{N}^{\star}}(M, x)\right| x_{x^{e}}\right)$ $+\omega(N-M) \leq \gamma_{V}\left(\sigma_{\delta}(P)\right)+\omega(N-M)$ and thus (i).

(ii) From the dynamic programming equation (4) and $u \equiv u^{e}$ we obtain

$$
V_{N}\left(x^{e}\right) \leq M \ell\left(x^{e}, u^{e}\right)+V_{N-M}\left(x^{e}\right) .
$$

On the other hand, from (5) we have

$$
\begin{aligned}
& V_{N}\left(x^{e}\right)= J_{M}\left(x, u_{N}^{\star e}\right)+V_{N-M}\left(x_{u_{N}^{\star e}}\left(M, x^{e}\right)\right) \\
&= \underbrace{\widetilde{J_{M}}\left(x, u_{N}^{\star e}\right)}_{\geq 0}-\lambda\left(x^{e}\right)+\lambda\left(x_{u_{N}^{\star e}}\left(M, x^{e}\right)\right)+M \ell\left(x^{e}, u^{e}\right)+V_{N-M}\left(x_{u_{N}^{\star e}}\left(M, x^{e}\right)\right) \\
& \geq V_{N-M}\left(x^{e}\right)+M \ell\left(x^{e}, u^{e}\right)+\left[V_{N-M}\left(x_{u_{N}^{\star e}}\left(M, x^{e}\right)\right)-V_{N-M}\left(x^{e}\right)\right] \\
&+\left[\lambda\left(x_{u_{N}^{\star e}}\left(M, x^{e}\right)\right)-\lambda\left(x^{e}\right)\right]
\end{aligned}
$$

Now since $V_{N-M}$ and $\lambda$ satisfy Assumption 12 (ii) and $x_{u_{N}^{\star e}}\left(M, x^{e}\right) \approx_{P} x^{e}$ for all $M \notin$ $\mathscr{Q}\left(x^{e}, u_{N}^{\star e}, P, N\right)$, we can conclude that the differences in the squared brackets have values $\approx_{S} 0$ which shows the assertion.

(iii) Fix $x \in \mathbb{X}$ and let $u_{N}^{\star}$ and $\tilde{u}_{N}^{\star} \in \mathbb{U}^{N}(x)$ denote the optimal control minimizing $J_{N}(x, u)$ and $\widetilde{J}_{N}(x, u)$, respectively. We note that if the optimal control problem with cost $\ell$ is strictly dissipative then the problem with cost $\tilde{\ell}$ is strictly dissipative, too, with bounded storage function $\lambda \equiv 0$ and same $\rho \in \mathscr{K}_{\infty}$. Moreover, $V_{N}(x) \leq$ $N \ell\left(x^{e}, u^{e}\right)+\gamma_{V}\left(|x|_{x^{e}}\right)+\omega(N)$ and $\widetilde{V}_{N}(x) \leq N \tilde{\ell}\left(x^{e}, u^{e}\right)+\gamma_{\tilde{V}}\left(|x|_{x^{e}}\right)$, since $V_{N}\left(x^{e}\right) \leq$ 
$N \ell\left(x^{e}, u^{e}\right)$ and $\widetilde{V}_{N}\left(x^{e}\right)=0$. Hence, the turnpike property from Remark 11(ii) applies to the optimal trajectories for both problems, yielding $\sigma_{\delta} \in \mathscr{L}$ and $\mathscr{Q}\left(x, u_{N}^{\star}, P, N\right)$ for $x_{u_{N}^{\star}}$ and $\tilde{\sigma}_{\tilde{\delta}}$ and $\widetilde{\mathscr{Q}}\left(x, \tilde{u}_{N}^{\star}, P, N\right)$ for $x_{\tilde{u}_{N}^{\star}}$. For all $M \notin \widetilde{\mathscr{Q}}\left(x, \tilde{u}_{N}^{\star}, P, N\right) \cup \mathscr{Q}\left(x^{e}, u_{N}^{\star e}, P, N\right)$ we can estimate

$$
\begin{aligned}
V_{N}(x) \leq & J_{M}\left(x, \tilde{u}_{N}^{\star}\right)+V_{N-M}\left(x_{\tilde{u}_{N}^{\star}}(M)\right) \\
\leq & J_{M}\left(x, \tilde{u}_{N}^{\star}\right)+V_{N-M}\left(x^{e}\right)+\gamma_{V}\left(\tilde{\sigma}_{\tilde{\delta}}(P)\right)+\omega(N-M) \\
\leq & \widetilde{J}_{M}\left(x, \tilde{u}_{N}^{\star}\right)-\lambda(x)+\lambda\left(x^{e}\right)+M \ell\left(x^{e}, u^{e}\right)+V_{N-M}\left(x^{e}\right)+\gamma_{V}\left(\tilde{\sigma}_{\tilde{\delta}}(P)\right) \\
& \quad+\gamma_{\lambda}\left(\tilde{\sigma}_{\tilde{\delta}}(P)\right)+\omega(N-M) \\
\lesssim_{S} & \widetilde{V}_{N}(x)-\lambda(x)+V_{N}\left(x^{e}\right)
\end{aligned}
$$

for $S=\min \{P, N-M\}$, where we have applied the dynamic programming equation (4) in the first inequality, the turnpike property for $x_{\tilde{u}_{N}^{\star}}$ and Assumption 12 and (29) in the second and third inequality and (i) applied to $\widetilde{V}_{N}$, and (ii) applied to $\ell$ in the last step. Moreover, $\lambda\left(x^{e}\right)=0$ and $\widetilde{V}_{N}\left(x^{e}\right)=0$ were used.

By exchanging the two optimal control problems and using the same inequalities as above, we get

$$
\widetilde{V}_{N}(x) \lesssim_{S} V_{N}(x)+\lambda(x)-V_{N}\left(x^{e}\right)
$$

for all $M \notin \mathscr{Q}\left(x, u_{N}^{\star}, P, N\right) \cup \widetilde{\mathscr{Q}}\left(x^{e}, \tilde{u}_{N}^{\star e}, P, N\right)$. Together this implies

$$
\widetilde{V}_{N}(x) \approx_{S} V_{N}(x)+\lambda(x)-V_{N}\left(x^{e}\right)
$$

for all $M \notin \mathscr{Q}\left(x, u_{N}^{\star}, P, N\right) \cup \widetilde{\mathscr{Q}}\left(x, u_{N}^{\star}, P, N\right) \cup \mathscr{Q}\left(x, u_{N}^{\star e}, P, N\right) \cup \widetilde{\mathscr{Q}}\left(x^{e}, \tilde{u}_{N}^{\star e}, P, N\right)$ and $S=$ $\min \{P, N-M\}$.

Now, choosing $P=\lfloor N / 5\rfloor$, the union of the four $\mathscr{Q}$-sets has at most $4 N / 5$ elements, hence there exists $M \leq N / 5$ for which this approximate inequality holds. This yields $S=\lfloor N / 5\rfloor$ and thus $\approx_{S}$ implies $\approx_{N}$, which shows (ii).

We note that precise quantitative statements can be made for the error terms "hiding" in the $\approx_{J}$-notation. Essentially, these terms depend on the distance between the optimal trajectories to the optimal equilibrium in the turnpike property, as measured by the function $\sigma_{\delta}$ in Remark 11(ii), and by the functions from Assumption 12. For details we refer to [14, Chapter 8].

Now, as in the previous section we can proceed in two different ways. Again, the first way consists in assuming $\ell\left(x^{e}, u^{e}\right)=0$ and the infinite horizon problem is well defined, implying that $\left|V_{\infty}(x)\right|$ is finite for all $x \in \mathbb{X}$. In this case, we can derive the following additional relations.

Lemma 14 Let $\mathbb{X}$ be bounded, let Assumption 12 hold and assume $\ell\left(x^{e}, u^{e}\right)=0$. Then the following approximate equalities hold.

$$
\begin{array}{rrlr}
V_{\infty}(x) & \approx_{P} & J_{M}\left(x, u_{\infty}^{\star}\right)+V_{\infty}\left(x^{e}\right) & \text { for all } M \notin \mathscr{Q}\left(x, u_{\infty}^{\star}, P, \infty\right) \\
J_{M}\left(x, u_{\infty}^{\star}\right) & \approx_{S} & J_{M}\left(x, u_{N}^{\star}\right) & \text { for all } M \notin \mathscr{Q}\left(x, u_{N}^{\star}, P, N\right) \\
& & \cup \mathscr{Q}\left(x, u_{\infty}^{\star}, P, \infty\right) .
\end{array}
$$


Here $P \in \mathbb{N}$ is an arbitrary number, $S:=\min \{P, N-M\}$ and $u_{\infty}^{\star}$ and $u_{N}^{\star}$ are the controls minimizing $J_{\infty}(x, u)$ and $J_{N}(x, u)$, respectively.

Proof. (i) The infinite horizon dynamic programming equation (13) yields

$$
V_{\infty}(x)=J_{M}\left(x, u_{\infty}^{\star}\right)+V_{\infty}\left(x_{u_{\infty}^{\star}}(M, x)\right) .
$$

Hence, we obtain

$$
V_{\infty}(x)=J_{M}\left(x, u_{\infty}^{\star}\right)+V_{\infty}\left(x^{e}\right)+\left[V_{\infty}\left(x_{u_{\infty}^{\star}}(M, x)\right)-V_{\infty}\left(x^{e}\right)\right] .
$$

From the turnpike property in Remark 11(ii) and Assumption 12 for $N=\infty$ we obtain that the term in square brackets is $\approx_{P} 0$ for all $M \notin \mathscr{Q}\left(x, u_{\infty}^{\star}, P, \infty\right)$, which shows (i).

(ii) The finite horizon dynamic programming equations (4) and (5) imply that $u=u_{N}^{\star}$ minimizes the expression $J_{M}(x, u)+V_{N-M}\left(x_{u}(M, x)\right)$. Using the turnpike property and Assumption 12(ii) for $V_{N}$ this yields

$$
\begin{aligned}
& J_{M}\left(x, u_{N}^{\star}\right)+V_{N-M}\left(x^{e}\right) \approx_{S} J_{M}\left(x, u_{N}^{\star}\right)+V_{N-M}\left(x_{u_{N}^{\star}}(M, x)\right) \\
& \quad \leq J_{M}\left(x, u_{\infty}^{\star}\right)+V_{N-M}\left(x_{u_{\infty}^{\star}}(M, x)\right) \approx_{S} J_{M}\left(x, u_{\infty}^{\star}\right)+V_{N-M}\left(x^{e}\right) .
\end{aligned}
$$

for all $M \notin \mathscr{Q}\left(x, u_{N}^{\star}, P, N\right)$ and $S=\min \{P, N-M\}$.

Conversely, the infinite horizon dynamic programming equations (12) and (13) imply that $u_{\infty}^{\star}$ minimizes the expression $J_{M}\left(x, u_{\infty}^{\star}\right)+V_{\infty}\left(x_{u_{\infty}^{\star}}(M, x)\right)$. Using the turnpike property and Assumption 12(ii) for $V_{\infty}$ this yields

$$
\begin{aligned}
& J_{M}\left(x, u_{\infty}^{\star}\right)+V_{\infty}\left(x^{e}\right) \approx_{P} J_{M}\left(x, u_{\infty}^{\star}\right)+V_{\infty}\left(x_{u_{\infty}^{\star}}(M, x)\right) \\
& \quad \leq J_{M}\left(x, u_{N}^{\star}\right)+V_{\infty}\left(x_{u_{N}^{\star}}(M, x)\right) \approx_{P} J_{M}\left(x, u_{N}^{\star}\right)+V_{\infty}\left(x^{e}\right)
\end{aligned}
$$

for all $M \notin \mathscr{Q}\left(x, u_{\infty}^{\star}, P, \infty\right)$. Combining these two approximate inequalities then implies (ii).

With these preparations we can state our first theorem on the performance of economic MPC without terminal conditions.

Theorem 15. Consider the MPC scheme without terminal conditions satisfying Assumption 12 and let $\mathbb{X}$ be bounded. Then there is $\delta_{1} \in \mathscr{L}$ such that for all $x \in \mathbb{X}$ the closed loop solution $x_{M P C}(k)$ generated by this scheme with $x_{M P C}(0)=x$ satisfies the inequality

$$
J_{K}^{c l}\left(x, \mu_{N}\right)+V_{\infty}\left(x_{M P C}(K)\right) \leq V_{\infty}(x)+K \delta_{1}(N)
$$

for all $K, N \in \mathbb{N}$.

Proof. We pick $x \in \mathbb{X}$ and abbreviate $x^{+}:=f\left(x, \mu_{N}(x)\right)$. For the corresponding optimal control $u_{N}^{\star}$, the relation (6) yields that $u_{N}^{\star}(\cdot+1)$ is an optimal control for initial value $x^{+}$and horizon $N-1$. Hence, for each $M \in\{1, \ldots, N\}$ we obtain 


$$
\begin{aligned}
\ell\left(x, \mu_{N}(x)\right)=V_{N}(x)-V_{N-1}\left(x^{+}\right) & =J_{N}\left(x, u_{N}^{\star}\right)-J_{N-1}\left(x^{+}, u_{N}^{\star}(\cdot+1)\right) \\
& =J_{M}\left(x, u_{N}^{\star}\right)-J_{M-1}\left(x^{+}, u_{N}^{\star}(\cdot+1)\right)
\end{aligned}
$$

where the last equality follows from the fact that the omitted terms in the sums defining $J_{M}\left(x, u_{N}^{\star}\right)$ and $J_{M-1}\left(x^{+}, u_{N}^{\star}(\cdot+1)\right)$ coincide. Using Lemma 14(i) for $N, x$ and $M$ and for $N-1, x^{+}$and $M-1$, respectively, yields

$$
\begin{aligned}
V_{\infty}(x)-V_{\infty}\left(x^{+}\right) & \approx_{P} J_{M}\left(x, u_{\infty}^{\star}\right)+V_{\infty}\left(x^{e}\right)-J_{M-1}\left(x^{+}, u_{\infty}^{\star}\right)-V_{\infty}\left(x^{e}\right) \\
& \approx_{P} J_{M}\left(x, u_{\infty}^{\star}\right)-J_{M-1}\left(x^{+}, u_{\infty}^{\star}\right) .
\end{aligned}
$$

Putting the two (approximate) equations together and using Lemma 14(ii) yields

$$
\ell\left(x, \mu_{N}(x)\right) \approx_{S} V_{\infty}(x)-V_{\infty}\left(x^{+}\right) .
$$

for all $M \in\{1, \ldots, N\}$ satisfying $M \notin \mathscr{Q}\left(x, u_{N}^{\star}, P, N\right) \cup \mathscr{Q}\left(x, u_{\infty}^{\star}, P, \infty\right)$ and $M-1 \notin$ $\mathscr{Q}\left(x^{+}, u_{N}^{\star}(\cdot+1), P, N-1\right) \cup \mathscr{Q}\left(x^{+}, u_{\infty}^{\star}(\cdot+1), P, \infty\right)$. Since each of the four $\mathscr{Q}$ sets contains at most $P$ elements, their union contains at most $4 P$ elements and hence if $N>8 P$ then there is at least one such $M$ with $M \leq N / 2$.

Thus, choosing $P=\lfloor(N-1) / 8\rfloor$ yields the existence of $M \leq N / 2$ such that (31) holds with $S=\lfloor(N-1) / 8\rfloor$, implying that $\approx_{S}$ in $(31)$ can be replaced by $\approx_{N}$. Hence, the error in (31) can be bounded by $\delta_{1}(N)$ for a function $\delta_{1} \in \mathscr{L}$, yielding

$$
\ell\left(x, \mu_{N}(x)\right) \leq V_{\infty}(x)-V_{\infty}\left(x^{+}\right)+\delta_{1}(N)
$$

Applying (32) for $x=x_{M P C}(k), k=0, \ldots, K-1$, we can then conclude

$$
\begin{aligned}
J_{K}^{c l}\left(x, \mu_{N}\right) & =\sum_{k=0}^{K-1} \ell\left(x_{M P C}(k), \mu_{N}\left(x_{M P C}(k)\right)\right) \\
& \leq \sum_{k=0}^{K-1}\left(V_{\infty}\left(x_{M P C}(k)\right)-V_{\infty}\left(x_{M P C}(k+1)\right)+\delta_{1}(N)\right) \\
& \leq V_{\infty}(x)-V_{\infty}\left(x_{M P C}(K)\right)+K \delta_{1}(N)
\end{aligned}
$$

This proves the claim.

The interpretation of inequality (30) is as follows: If we concatenate the closed loop trajectory $\left(x_{M P C}(0), \ldots, x_{M P C}(K)\right)$ with the infinite horizon optimal trajectory emanating from $x_{M P C}(K)$, then the overall cost $J_{K}^{c l}\left(x, \mu_{N}\right)+V_{\infty}\left(x_{M P C}(K)\right)$ is less than the optimal cost $V_{\infty}(x)$ plus the error term $K \delta_{1}(N)$. In other words, for large $N$ the initial piece of the MPC closed loop trajectory is an initial piece of an approximately optimal infinite horizon trajectory.

With similar arguments as in the proofs of Lemma 13 and 14 one can also prove the approximate equation

$$
V_{N}(x) \approx_{N} V_{N-1}(x)+\ell\left(x^{e}, u^{e}\right)
$$


Using this relation, Lemma 13(iii) and the dynamic programming equation (7), for $x^{+}=f\left(x, \mu_{N}(x)\right)$ we obtain

$$
\begin{aligned}
\widetilde{V}_{N}\left(x^{+}\right) & \approx_{N} V_{N}\left(x^{+}\right)+\lambda\left(x^{+}\right)-V_{N}\left(x^{e}\right) \\
& \approx_{N} V_{N-1}\left(x^{+}\right)+\ell\left(x^{e}, u^{e}\right)+\lambda\left(x^{+}\right)-V_{N}\left(x^{e}\right) \\
& =V_{N}(x)-\ell\left(x, \mu_{N}(x)\right)+\ell\left(x^{e}, u^{e}\right)+\lambda\left(x^{+}\right)-V_{N}\left(x^{e}\right) \\
& \approx_{N} \widetilde{V}_{N}(x) \underbrace{-\ell\left(x, \mu_{N}(x)\right)+\ell\left(x^{e}, u^{e}\right)+\lambda\left(x^{+}\right)-\lambda(x)}_{=-\tilde{\ell}\left(x, \mu_{N}(x)\right)} .
\end{aligned}
$$

This implies that the modified optimal value function decays in each step, except for an error term which vanishes as $N \rightarrow \infty$. Since $\widetilde{V}_{N}(x) \geq \rho\left(|x|_{x^{e}}\right)$ and $\tilde{\ell}\left(x, \mu_{N}(x)\right) \geq$ $\rho\left(|x|_{x^{e}}\right)$, from this we can conclude that as $k \rightarrow \infty$ the closed loop solution $x_{M P C}(k)$ converges to a neighbourhood of $x^{e}$, which shrinks down to $x^{e}$ for $N \rightarrow \infty$ (for a rigorous application of this argument see [14, Section 8.6]). In fact, due to the upper bound on $\widetilde{V}_{N}$ induced by Assumption 12(ii), we can even conclude the existence of $\beta \in \mathscr{K} \mathscr{L}$ and $\kappa \in \mathscr{L}$ such that for all $x \in \mathbb{X}$ the MPC closed loop solution $x_{M P C}(k)$ with $x_{M P C}(0)=x$ satisfies

$$
\left|x_{M P C}(k)\right|_{x^{e}} \leq \max \left\{\beta\left(|x|_{x^{e}}, k\right), \kappa(N)\right\}
$$

for all $N, k \in \mathbb{N}$, cf. [14, Theorem 8.33]. This means that the optimal equilibrium $x^{e}$ is practically asymptotically stable for the MPC closed loop.

We note that already in very simple examples (see again $[11,12]$ or $[14$, Chapter 8]) convergence to the optimal equilibrium $x^{e}$ will not hold for the MPC closed loop. Hence, in the absence of terminal conditions, practical asymptotic stability of $x^{e}$ is in general the best one can obtain. This also explains the factor $K$ before $\delta_{1}(N)$ in the estimate from Theorem 15. Since the trajectory always has a little distance to the optimal equilibrium, in each step we collect a small error and these errors sum up from 0 to $K-1$, resulting in the factor $K$ in front of the error term. Note, however, that the fact that the trajectory stays near $x^{e}$ prevents the solution from deteriorating as $k \rightarrow \infty$, even though the error term in (30) tends to infinite for large $K$.

Due to the fact that the closed loop solution converges to a neighbourhood of $x^{e}$, it seems plausible that also without terminal conditions we can obtain a performance estimate for $J_{K}^{c l}\left(x, \mu_{N}\right)$ without reference to the infinite horizon problem, similar to (28). Our last theorem shows that this is indeed possible.

Theorem 16. Consider the MPC scheme without terminal conditions satisfying Assumption 12 and let $\mathbb{X}$ be bounded. Then there are $\delta_{1}, \delta_{2}, \delta_{3} \in \mathscr{L}$ such that for all $x \in \mathbb{X}$ the closed loop solution $x_{M P C}(k)$ generated by this scheme with $x_{M P C}(0)=x$ satisfies the inequality

$$
J_{K}^{c l}\left(x, \mu_{N}\right) \leq \inf _{u \in \mathbb{U}_{\delta(K)}^{K}(x)} J_{K}(x, u)+\delta_{1}(N)+K \delta_{2}(N)+\delta_{3}(K)
$$

for all $K, N \in \mathbb{N}$, for $\mathbb{U}_{\delta(K)}^{K}(x)$ from (27) with $\delta(K):=\left|x_{M P C}(K)\right|_{x}$. 
Proof. From (33) we obtain

$$
\tilde{\ell}\left(x, \mu_{N}(x)\right) \approx_{N} \widetilde{V}_{N}(x)-\widetilde{V}_{N}\left(f\left(x, \mu_{N}(x)\right)\right) .
$$

We denote the error in this approximate equation by $\delta_{2}(N)$. Summing $\tilde{\ell}\left(x, \mu_{N}(x)\right)$ along the closed-loop trajectory then yields

$$
\sum_{k=0}^{K-1} \tilde{\ell}\left(x_{M P C}(k), \mu_{N}\left(x_{M P C}(k)\right)\right) \leq \widetilde{V}_{N}(x)-\widetilde{V}_{N}\left(x_{M P C}(K)\right)+K \delta_{2}(N) .
$$

Now the dynamic programming equation (4) and Assumption 12(ii) yield for all $K \in\{1, \ldots, N\}$ and all $u \in \mathbb{U}_{\delta(K)}^{K}(x)$

$$
\widetilde{J}_{K}(x, u)=\underbrace{\widetilde{J}_{K}(x, u)+\widetilde{V}_{N-K}\left(x_{u}(K, x)\right)}_{\geq \widetilde{V}_{N}(x)}-\underbrace{\widetilde{V}_{N-K}\left(x_{u}(K, x)\right)}_{\leq \gamma_{\tilde{V}}(\delta(K))} \geq \widetilde{V}_{N}(x)-\gamma_{\tilde{V}}(\delta(K)) .
$$

Due to the non-negativity of $\tilde{\ell}$, for $K \geq N$ we get $\widetilde{J}_{K}(x, u) \geq \widetilde{V}_{N}(x)$ for all $u \in \mathbb{U}^{K}(x)$. Hence (37) holds for all $K \in \mathbb{N}$. Moreover, we have $\widetilde{V}_{N}(x) \geq 0$. Using (36), (37), (29) and the definition of $\delta_{2}$, for all $u \in \mathbb{U}_{\delta(K)}^{K}(x)$ we obtain

$$
\begin{aligned}
& J_{K}^{c l}\left(x, \mu_{N}(x)\right)=\sum_{k=0}^{K-1} \tilde{\ell}\left(x_{M P C}(k), \mu_{N}\left(x_{M P C}(k)\right)\right)-\lambda(x)+\lambda\left(x_{M P C}(K)\right) \\
& \leq \widetilde{V}_{N}(x)-\widetilde{V}_{N}\left(x_{M P C}(K)\right)+K \delta_{2}(N)-\lambda(x)+\lambda\left(x_{M P C}(K)\right) \\
& \leq \widetilde{J}_{K}(x, u)+\gamma_{\tilde{V}}(\delta(K))-\widetilde{V}_{N}\left(x_{M P C}(K)\right)+K \delta_{2}(N)-\lambda(x)+\lambda\left(x_{M P C}(K)\right) \\
& =J_{K}(x, u)+\gamma_{\tilde{V}}(\delta(K))-\widetilde{V}_{N}\left(x_{M P C}(K)\right)+K \delta_{2}(N)-\lambda\left(x_{u}(K, x)\right)+\lambda\left(x_{M P C}(K)\right) \\
& \leq J_{K}(x, u)+\gamma_{\tilde{V}}(\delta(K))+K \delta_{2}(N)+2 \gamma_{\lambda}(\delta(K)) .
\end{aligned}
$$

Now from (34) we obtain

$$
\begin{aligned}
\gamma_{\widehat{V}}(\delta(K))+2 \gamma_{\lambda}(\delta(K)) \leq & \underbrace{\sup _{x \in \mathbb{X}} \gamma_{\widehat{V}}\left(\beta\left(|x|_{x^{e}}, K\right)\right)+2 \gamma_{\lambda}\left(\beta\left(|x|_{x^{e}}, K\right)\right)}_{=: \delta_{3}(K)} \\
& +\underbrace{\gamma_{\widehat{V}}(\kappa(N))+2 \gamma_{\lambda}(\kappa(N))}_{=: \delta_{1}(N)}
\end{aligned}
$$

which finishes the proof.

The interpretation of this result is similar to that of (28): among all solutions steering $x$ into the $\delta(K)$-neighbourhood of the optimal equilibrium $x^{e}$, MPC yields the cheapest one up to error terms vanishing for large $K$ and larger $N$.

We would like to note that the results from this section have been extended in various ways. For instance, in many examples it can be observed that the error terms $\delta_{j}(N)$ converge to 0 exponentially fast as $N \rightarrow \infty$, i.e., that they are of the form $\delta_{j}(N)=C \Theta^{N}$ for $C>0$ and $\Theta \in(0,1)$. Conditions under which this can be 
rigorously proved can be found in [18]. Another extension concerns replacing the optimal equilibrium $x^{e}$ by a periodic orbit. Corresponding results can be found, e.g., in $[2,24,31]$. Currently, one research focus is the extension of the results to arbitrary time varying problems, in which $x^{e}$ is replaced by a general time varying trajectory with certain optimality properties. First results on this topic will appear in [14].

\section{Conclusions}

We have presented a collection of results about the infinite horizon closed loop performance and stability of MPC closed loop trajectories, for both stabilizing and economic MPC and for schemes with and without terminal conditions. In the course of this analysis, we have shown that dynamic programming arguments are needed in a lot of different places and for various purposes. Dynamic programming thus forms an indispensable tool for understanding the behavior of MPC schemes.

\section{References}

1. Amrit, R., Rawlings, J.B., Angeli, D.: Economic optimization using model predictive control with a terminal cost. Annual Rev. Control 35, 178-186 (2011)

2. Angeli, D., Amrit, R., Rawlings, J.B.: On average performance and stability of economic model predictive control. IEEE Trans. Autom. Control 57(7), 1615-1626 (2012)

3. Angeli, D., Rawlings, J.B.: Receding horizon cost optimization and control for nonlinear plants. In: Proceedings of the 8th IFAC Symposium on Nonlinear Control Systems - NOLCOS 2010, pp. 1217-1223. Bologna, Italy (2010)

4. Bellman, R.: Dynamic programming. Princeton Landmarks in Mathematics. Princeton University Press, Princeton, NJ (2010). Reprint of the 1957 edition

5. Bertsekas, D.P.: Dynamic Programming and Optimal Control. Vol. 1 and 2. Athena Scientific, Belmont, MA (1995)

6. Camacho, E.F., Bordons, C.: Model predictive control, 2nd edn. Springer-Verlag, London (2004)

7. Chen, H., Allgöwer, F.: A quasi-infinite horizon nonlinear model predictive control scheme with guaranteed stability. Automatica 34(10), 1205-1217 (1998)

8. Diehl, M., Amrit, R., Rawlings, J.B.: A Lyapunov function for economic optimizing model predictive control. IEEE Trans. Autom. Control 56, 703-707 (2011)

9. Faulwasser, T., Bonvin, D.: On the design of economic NMPC based on approximate turnpike properties. In: Proceedings of the 54th IEEE Conference on Decision and Control - CDC 2015, pp. 4964-4970 (2015)

10. Forbes, M.G., Patwardhan, R.S., Hamadah, H., Gopaluni, R.B.: Model predictive control in industry: Challenges and opportunities. In: Proceedings of the 9th IFAC Symposium on Advanced Control of Chemical Processes - ADCHEM 2015, IFAC-PapersOnLine, vol. 48, pp. 531-538. Whistler, Canada (2015)

11. Grüne, L.: Economic receding horizon control without terminal constraints. Automatica 49(3), 725-734 (2013)

12. Grüne, L.: Approximation properties of receding horizon optimal control. Jahresber. DMV 118(1), 3-37 (2016) 
13. Grüne, L., Panin, A.: On non-averaged performance of economic MPC with terminal conditions. In: Proceedings of the 54th IEEE Conference on Decision and Control - CDC 2015, pp. 4332-4337. Osaka, Japan (2015)

14. Grüne, L., Pannek, J.: Nonlinear Model Predictive Control. Theory and Algorithms, 2nd edn. Springer-Verlag, London (2017)

15. Grüne, L., Pannek, J., Seehafer, M., Worthmann, K.: Analysis of unconstrained nonlinear MPC schemes with time varying control horizon. SIAM J. Control Optim. 48, 4938-4962 (2010)

16. Grüne, L., Pirkelmann, S.: Closed-loop performance analysis for economic model predictive control of time-varying systems. In: Proceedings of the 56th IEEE Conference on Decision and Control - CDC 2017. Melbourne, Australia (2017). To appear

17. Grüne, L., Semmler, W.: Using dynamic programming with adaptive grid scheme for optimal control problems in economics. J. Econ. Dyn. Control 28, 2427-2456 (2004)

18. Grüne, L., Stieler, M.: Asymptotic stability and transient optimality of economic MPC without terminal conditions. J. Proc. Control 24(8), 1187-1196 (2014)

19. Keerthi, S.S., Gilbert, E.G.: Optimal infinite horizon feedback laws for a general class of constrained discrete-time systems: stability and moving horizon approximations. J. Optimiz. Theory Appl. 57, 265-293 (1988)

20. Kerrigan, E.C.: Robust constraint satisfaction: Invariant sets and predictive control. PhD Thesis, University of Cambridge (2000)

21. Lincoln, B., Rantzer, A.: Relaxing dynamic programming. IEEE Trans. Autom. Control 51, 1249-1260 (2006)

22. Magni, L., Sepulchre, R.: Stability margins of nonlinear receding-horizon control via inverse optimality. Systems Control Lett. 32(4), 241-245 (1997)

23. Mayne, D.Q., Rawlings, J.B., Rao, C.V., Scokaert, P.O.M.: Constrained model predictive control: stability and optimality. Automatica 36, 789-814 (2000)

24. Müller, M.A., Grüne, L.: Economic model predictive control without terminal constraints for optimal periodic behavior. Automatica 70, 128-139 (2016)

25. de Nicolao, G., Magni, L., Scattolini, R.: Stabilizing receding-horizon control of nonlinear time-varying systems. IEEE Trans. Automat. Contr. 43, 1030-1036 (1998)

26. Olanrewaju, O.I., Maciejowski, J.M.: Implications of dissipativity on stability of economic model predictive control - the indefinite linear quadratic case. Syst. Contr. Lett. 100, 43-50 (2017)

27. Primbs, J.A., Nevistić, V.: Feasibility and stability of constrained finite receding horizon control. Automatica 36(7), 965-971 (2000)

28. Rawlings, J.B., Mayne, D.Q.: Model Predictive Control: Theory and Design. Nob Hill Publishing, Madison, Wisconsin (2009)

29. Tuna, S.E., Messina, M.J., Teel, A.R.: Shorter horizons for model predictive control. In: Proceedings of the 2006 American Control Conference, pp. 863-868. Minneapolis, Minnesota, USA (2006)

30. Worthmann, K.: Stability Analysis of Unconstrained Receding Horizon Control Schemes. $\mathrm{PhD}$ Thesis, Universität Bayreuth (2011)

31. Zanon, M., Grüne, L., Diehl, M.: Periodic optimal control, dissipativity and MPC. IEEE Trans. Auto. Cont. 62(6), 2943-2949 (2017) 